\title{
Certain partial differential subordinations on some Reinhardt domains in $\mathbb{C}^{n}$
}

\author{
by Gabriela Kohr and Mirela Kohr (Cluj)
}

\begin{abstract}
We obtain an extension of Jack-Miller-Mocanu's Lemma for holomorphic mappings defined in some Reinhardt domains in $\mathbb{C}^{n}$. Using this result we consider first and second order partial differential subordinations for holomorphic mappings defined on the Reinhardt domain $B_{2 p}$ with $p \geq 1$.
\end{abstract}

1. Preliminaries. Let $\mathbb{C}^{n}$ denote the space of $n$ complex variables $z=$ $\left(z_{1}, \ldots, z_{n}\right)^{\prime}$ with the euclidian inner product

$$
\langle z, w\rangle=\sum_{i=1}^{n} z_{i} \bar{w}_{i}
$$

and the norm $\|z\|=\sqrt{\langle z, z\rangle}$, for all $z, w \in \mathbb{C}^{n}$.

The open euclidian ball $\left\{z \in \mathbb{C}^{n}:\|z\|<r\right\}$ is denoted by $B_{r}$ and the open unit euclidian ball is abbreviated by $B_{1}=B$. For $n=1$, the open ball $B_{r}$ is denoted by $U_{r}$ and the open unit disc is abbreviated by $U_{1}=U$. The Reinhardt domain

$$
\left\{z \in \mathbb{C}^{n}: \sum_{i=1}^{n}\left|z_{i}\right|^{2 p}<r\right\}
$$

is denoted by $B_{2 p}(r)$, where $p \geq 1$, and the Reinhardt domain $B_{2 p}(1)$ is abbreviated by $B_{2 p}(1)=B_{2 p}$.

The origin $(0, \ldots, 0)^{\prime}$ is always denoted by 0 .

As usual, by $L\left(\mathbb{C}^{n}, \mathbb{C}^{m}\right)$ we denote the space of all continuous linear operators from $\mathbb{C}^{n}$ into $\mathbb{C}^{m}$ with the standard operator norm. The letter $I$ will always represent the identity operator in $L\left(\mathbb{C}^{n}, \mathbb{C}^{n}\right)$.

The class of holomorphic mappings from a domain $G \subseteq \mathbb{C}^{n}$ into $\mathbb{C}^{n}$ is denoted by $H(G)$.

1991 Mathematics Subject Classification: 32H02, 30C45.

Key words and phrases: subordination, biholomorphic mapping, Reinhardt domain. 
A mapping $f \in H(G)$ is said to be locally biholomorphic on $G$ if its Fréchet derivative

$$
D f(z)=\left(\frac{\partial f_{j}}{\partial z_{k}}(z)\right)_{1 \leq j, k \leq n}
$$

as an element of $L\left(\mathbb{C}^{n}, \mathbb{C}^{n}\right)$ is nonsingular at each point $z \in G$.

A mapping $f \in H(G)$ is called biholomorphic on $G$ if the inverse mapping $f^{-1}$ exists, is holomorphic on a domain $\Omega$ and $f^{-1}(\Omega)=G$.

Let $D^{2} f(z)$ be the Fréchet derivative of second order for $f \in H(G)$; then $D^{2} f(z)$ is a continuous bilinear operator from $\mathbb{C}^{n} \times \mathbb{C}^{n}$ into $\mathbb{C}^{n}$ and its restriction $D^{2} f(z)(u, \cdot)$ to $u \times \mathbb{C}^{n}$ belongs to $L\left(\mathbb{C}^{n}, \mathbb{C}^{n}\right)$.

The symbol "'" means the transpose of elements and matrices defined on $\mathbb{C}^{n}$. If $A=\left(a_{i j}\right)_{1 \leq i, j \leq n}$, then $\bar{A}=\left(\bar{a}_{i j}\right)_{1 \leq i, j \leq n}$, where $a_{i j} \in \mathbb{C}$.

If $\varphi$ is a $C^{2}$ complex function defined on a domain $\Omega \subset \mathbb{C}^{n}$, then we use the following notations:

$$
\begin{gathered}
\frac{\partial \varphi}{\partial z}(z)=\left(\frac{\partial \varphi}{\partial z_{1}}(z), \ldots, \frac{\partial \varphi}{\partial z_{n}}(z)\right)^{\prime} \\
\frac{\partial^{2} \varphi}{\partial z^{2}}(z)=\left[\frac{\partial^{2} \varphi}{\partial z_{i} \partial z_{j}}(z)\right]_{1 \leq i, j \leq n}, \quad \frac{\partial^{2} \varphi}{\partial \bar{z} \partial z}(z)=\left[\frac{\partial^{2} \varphi}{\partial \bar{z}_{i} \partial z_{j}}(z)\right]_{1 \leq i, j \leq n}
\end{gathered}
$$

for all $z=\left(z_{1}, \ldots, z_{n}\right)^{\prime} \in \Omega$.

If $z=\left(z_{1}, \ldots, z_{n}\right)^{\prime} \in \mathbb{C}^{n}$ and $p \geq 1$, let

$$
\|z\|_{2 p}=\left[\sum_{j=1}^{n}\left|z_{j}\right|^{2 p}\right]^{1 / 2 p} .
$$

Very recently the authors of [KO2] obtained an extension of Jack-MillerMocanu's Lemma [MM1] for holomorphic mappings defined on the unit ball of $\mathbb{C}^{n}$ with the supremum norm, and also extended the same result to holomorphic mappings defined on the unit ball with an arbitrary norm. Applications to subordinations were considered.

Another extension of Jack-Miller-Mocanu's Lemma was given in [KO4] for holomorphic mappings defined on some domains in $\mathbb{C}^{n}$ whose boundary is a real $C^{2}$ hypersurface. Using this result, the author of [KO1] gave some applications to subordinations of holomorphic mappings defined on some bounded domains for which the kernel function becomes infinite on the boundary.

In this paper we continue these investigations, giving another extension of Jack-Miller-Mocanu's Lemma to holomorphic mappings defined on the Reinhardt domain $B_{2 p}$ and we obtain some partial differential subordinations of first and second kind on $B_{2 p}$ with $p \geq 1$.

We shall use the following result. 
LEMMA 1.1 [MM1], [MM2]. Let $g(z)=a_{n} z^{n}+a_{n+1} z^{n+1}+\ldots$ be continuous on $\bar{U}_{r_{0}}$, and holomorphic on $U_{r_{0}} \cup\left\{z_{0}\right\}$, where $z_{0}=r_{0} e^{i \theta_{0}}, r_{0}<1$ and $n \geq 1$. If

$$
\left|g\left(z_{0}\right)\right|=\max \left\{|g(z)|:|z| \leq r_{0}\right\}
$$

then

$$
z_{0} g^{\prime}\left(z_{0}\right)=m g\left(z_{0}\right)
$$

and

$$
\operatorname{Re}\left[1+z_{0} g^{\prime \prime}\left(z_{0}\right) / g^{\prime}\left(z_{0}\right)\right] \geq m,
$$

where $m \geq n \geq 1$.

\section{Main results}

Theorem 2.1. Let $r \in(0,1), z_{0} \in \bar{B}_{2 p}(r)$, where $p \geq 1$, let $f \in H\left(B_{2 p}\right)$ with $f(0)=0, f$ locally biholomorphic at $z_{0}$ and $f \not \equiv 0$. If $z_{0}$ is defined by

$$
\left\|f\left(z_{0}\right)\right\|_{2 p}=\max \left\{\|f(z)\|_{2 p}: z \in \bar{B}_{2 p}(r)\right\},
$$

then there exists $0<s \leq\left[\left\|z_{0}\right\|_{2 p} /\left\|f\left(z_{0}\right)\right\|_{2 p}\right]^{2 p}$ such that

$$
\begin{aligned}
\left(\left|z_{1}^{0}\right|^{2(p-1)} \bar{z}_{1}^{0}, \ldots,\left|z_{n}^{0}\right|^{2(p-1)} \bar{z}_{n}^{0}\right)\left[D f\left(z_{0}\right)\right]^{-1} & \\
& =s\left(\left|f_{1}\left(z_{0}\right)\right|^{2(p-1)} \overline{f_{1}\left(z_{0}\right)}, \ldots,\left|f_{n}\left(z_{0}\right)\right|^{2(p-1)} \overline{f_{n}\left(z_{0}\right)}\right),
\end{aligned}
$$

where $z_{0}=\left(z_{1}^{0}, \ldots, z_{n}^{0}\right)^{\prime}$, and

$$
\begin{aligned}
\operatorname{Re}\left\{\left(D f\left(z_{0}\right) v\right)^{\prime}\right. & \left.\frac{\partial^{2} \varphi}{\partial w^{2}}\left(w_{0}\right) D f\left(z_{0}\right) v\right\} \\
+ & \left(\overline{\left.D f\left(z_{0}\right) v\right)^{\prime}} \frac{\partial^{2} \varphi}{\partial \bar{w} \partial w}\left(w_{0}\right) D f\left(z_{0}\right) v\right. \\
\leq & \frac{1}{s}\left\{\bar{v}^{\prime} \frac{\partial^{2} \varphi}{\partial \bar{z} \partial z}\left(z_{0}\right) v+\operatorname{Re}\left[v^{\prime} \frac{\partial^{2} \varphi}{\partial z^{2}}\left(z_{0}\right) v\right]\right. \\
& \left.-\operatorname{Re}\left[\left(\frac{\partial \varphi}{\partial z}\left(z_{0}\right)\right)^{\prime}\left[D f\left(z_{0}\right)\right]^{-1} D^{2} f\left(z_{0}\right)(v, v)\right]\right\}
\end{aligned}
$$

for all $v=\left(v_{1}, \ldots, v_{n}\right)^{\prime} \in \mathbb{C}^{n} \backslash\{0\}$, with $\operatorname{Re} \sum_{j=1}^{n} \bar{z}_{j}^{0} v_{j}\left|z_{j}^{0}\right|^{2(p-1)}=0$, where $\varphi(z)=\sum_{j=1}^{n}\left|z_{j}\right|^{2 p}$, for all $z=\left(z_{1}, \ldots, z_{n}\right)^{\prime} \in \mathbb{C}^{n}$ and $w_{0}=f\left(z_{0}\right)$.

Proof. Let $T_{z_{0}}\left(\partial B_{2 p}\left(r_{0}\right)\right)$ be the real tangent space to $\partial B_{2 p}\left(r_{0}\right)$ at $z_{0}$ and let $v \in T_{z_{0}}\left(\partial B_{2 p}\left(r_{0}\right)\right)$ be an arbitrary tangent vector, where $r_{0}=$ $\sum_{j=1}^{n}\left|z_{j}^{0}\right|^{2 p}$. Since $\partial B_{2 p}\left(r_{0}\right)$ is a $C^{2}$ hypersurface, it is well known that there exist an $\varepsilon>0$ and a twice differentiable curve $\gamma:(-\varepsilon, \varepsilon) \rightarrow \partial B_{2 p}\left(r_{0}\right)$ such that $\gamma(0)=z_{0}$ and $\frac{d \gamma}{d t}(0)=v$. Let $\varphi: \mathbb{C}^{n} \rightarrow \mathbb{R}$ be given by $\varphi(z)=$ 
$\sum_{j=1}^{n}\left|z_{j}\right|^{2 p}$ for all $z=\left(z_{1}, \ldots, z_{n}\right)^{\prime} \in \mathbb{C}^{n}$, and $\beta:(-\varepsilon, \varepsilon) \rightarrow \mathbb{R}$ by $\beta(t)=$ $(\varphi \circ f \circ \gamma)(t), t \in(-\varepsilon, \varepsilon)$. Then $\beta$ is a $C^{2}$ function on $(-\varepsilon, \varepsilon)$ and using (2.1) we obtain

$$
\beta(0)=\sum_{j=1}^{n}\left|f_{j}\left(z_{0}\right)\right|^{2 p}=\max \{\beta(t): t \in(-\varepsilon, \varepsilon)\} .
$$

Hence, $\beta^{\prime}(0)=0$ and $\beta^{\prime \prime}(0) \leq 0$.

On the other hand, a straightforward calculation yields

$$
\beta^{\prime}(t)=2 \operatorname{Re}\left\{\left(\frac{\partial \varphi}{\partial w}(w(t))\right)^{\prime} D f(\gamma(t)) \frac{d \gamma}{d t}(t)\right\}, \quad t \in(-\varepsilon, \varepsilon),
$$

where $w(t)=f(\gamma(t))$, so at $t=0$ we deduce that

$$
\begin{aligned}
0 & =\beta^{\prime}(0)=2 \operatorname{Re}\left\{\left(\frac{\partial \varphi}{\partial w}\left(f\left(z_{0}\right)\right)\right)^{\prime} D f\left(z_{0}\right) v\right\} \\
& =2 \operatorname{Re}\left\{p\left(\left|f_{1}\left(z_{0}\right)\right|^{2(p-1)} \overline{f_{1}\left(z_{0}\right)}, \ldots,\left|f_{n}\left(z_{0}\right)\right|^{2(p-1)} \overline{f_{n}\left(z_{0}\right)}\right) D f\left(z_{0}\right) v\right\} \\
& =2 p \operatorname{Re}\left\langle\left[\overline{D f\left(z_{0}\right)}\right]^{\prime}\left(\left|f_{1}\left(z_{0}\right)\right|^{2(p-1)} f_{1}\left(z_{0}\right), \ldots,\left|f_{n}\left(z_{0}\right)\right|^{2(p-1)} f_{n}\left(z_{0}\right)\right)^{\prime}, v\right\rangle .
\end{aligned}
$$

Since this relation is satisfied for every tangent vector $v$, we conclude that

$$
p\left[\overline{D f\left(z_{0}\right)}\right]^{\prime}\left(\left|f_{1}\left(z_{0}\right)\right|^{2(p-1)} f_{1}\left(z_{0}\right), \ldots,\left|f_{n}\left(z_{0}\right)\right|^{2(p-1)} f_{n}\left(z_{0}\right)\right)^{\prime}
$$

is a normal vector to $\partial B_{2 p}\left(r_{0}\right)$ at $z_{0}$, hence it is collinear with the outward normal vector to $\partial B_{2 p}\left(r_{0}\right)$ at $z_{0}$.

Let $N_{z_{0}}$ be the outward normal vector to $\partial B_{2 p}\left(r_{0}\right)$ at $z_{0}$. Then

$$
N_{z_{0}}=\frac{\partial \varphi}{\partial \bar{z}}\left(z_{0}\right)=p\left(\left|z_{1}^{0}\right|^{2(p-1)} z_{1}^{0}, \ldots,\left|z_{n}^{0}\right|^{2(p-1)} z_{n}^{0}\right)^{\prime} .
$$

Hence we can find a real number $\delta$ such that

$$
\begin{aligned}
\left(\left|f_{1}\left(z_{0}\right)\right|^{2(p-1)} f_{1}\left(z_{0}\right), \ldots,\left|f_{n}\left(z_{0}\right)\right|^{2(p-1)} f_{n}\left(z_{0}\right)\right)\left[\overline{D f\left(z_{0}\right)}\right] & \\
= & \delta\left(\left|z_{1}^{0}\right|^{2(p-1)} z_{1}^{0}, \ldots,\left|z_{n}^{0}\right|^{2(p-1)} z_{n}^{0}\right) .
\end{aligned}
$$

Now, since $f$ is locally biholomorphic at $z_{0}$, it follows that $\left[D f\left(z_{0}\right)\right]^{-1}$ exists and from (2.5) we conclude that $\delta \neq 0$ and

$$
\begin{aligned}
\left(\left|z_{1}^{0}\right|^{2(p-1)} \bar{z}_{1}^{0}, \ldots,\left|z_{n}^{0}\right|^{2(p-1)} \bar{z}_{n}^{0}\right)\left[D f\left(z_{0}\right)\right]^{-1} & \\
& =s\left(\left|f_{1}\left(z_{0}\right)\right|^{2(p-1)} \overline{f_{1}\left(z_{0}\right)}, \ldots,\left|f_{n}\left(z_{0}\right)\right|^{2(p-1)} \overline{f_{n}\left(z_{0}\right)}\right),
\end{aligned}
$$

where $s=1 / \delta$.

Consider the function

$$
g(\zeta)=\sum_{j=1}^{n} f_{j}\left(\zeta z_{0} /\left\|z_{0}\right\|_{2 p}\right)\left|f_{j}\left(z_{0}\right)\right|^{2(p-1)} \overline{f_{j}\left(z_{0}\right)}
$$


for all $\zeta \in \mathbb{C},|\zeta| \leq\left\|z_{0}\right\|_{2 p}$. Then $g \in H\left(U_{\left\|z_{0}\right\|_{2 p}} \cup\left\{\zeta_{0}\right\}\right) \cap C\left(\bar{U}_{\left\|z_{0}\right\|_{2 p}}\right)$, where $\zeta_{0}=\left\|z_{0}\right\|_{2 p}$. Also, $g(0)=0$ and, using Hölder's inequality, it is not difficult to see that

$$
\left|g\left(\zeta_{0}\right)\right|=\sum_{j=1}^{n}\left|f_{j}\left(z_{0}\right)\right|^{2 p}=\max \left\{|g(\zeta)|:|\zeta| \leq\left|\zeta_{0}\right|\right\} ;
$$

hence, from Lemma 1.1, there exists $m \geq 1$ such that

$$
\zeta_{0} g^{\prime}\left(\zeta_{0}\right)=m g\left(\zeta_{0}\right)
$$

On the other hand,

$$
\zeta_{0} g^{\prime}\left(\zeta_{0}\right)=\sum_{j=1}^{n}\left|f_{j}\left(z_{0}\right)\right|^{2(p-1)} \overline{f_{j}\left(z_{0}\right)} \sum_{k=1}^{n} \frac{\partial f_{j}}{\partial z_{k}}\left(z_{0}\right) z_{k}^{0},
$$

so, combining (2.6) and (2.7), we deduce that

$$
\begin{aligned}
\left(\left|f_{1}\left(z_{0}\right)\right|^{2(p-1)} \overline{f_{1}\left(z_{0}\right)}, \ldots,\left|f_{n}\left(z_{0}\right)\right|^{2(p-1)} \overline{f_{n}\left(z_{0}\right)}\right) & D f\left(z_{0}\right) z_{0} \\
& =m \sum_{j=1}^{n}\left|f_{j}\left(z_{0}\right)\right|^{2 p} .
\end{aligned}
$$

Multiplying both sides of (2.2) by $z=z_{0}$, and using (2.8), we obtain

$$
\frac{1}{s} \sum_{j=1}^{n}\left|z_{j}^{0}\right|^{2 p}=m \sum_{j=1}^{n}\left|f_{j}\left(z_{0}\right)\right|^{2 p} .
$$

Since $m \geq 1, z_{0} \neq 0$ and $f\left(z_{0}\right) \neq 0$, we deduce that

$$
0<s \leq \sum_{j=1}^{n}\left|z_{j}^{0}\right|^{2 p} / \sum_{j=1}^{n}\left|f_{j}\left(z_{0}\right)\right|^{2 p} .
$$

It remains to show (2.3). To do this we use (2.4) again. Differentiating it with respect to $t$, we obtain

$$
\begin{aligned}
\beta^{\prime \prime}(t)= & 2 \operatorname{Re}\left\{\left(D f(\gamma(t)) \frac{d \gamma}{d t}(t)\right)^{\prime} \frac{\partial^{2} \varphi}{\partial w^{2}}(w(t)) D f(\gamma(t)) \frac{d \gamma}{d t}(t)\right\} \\
& +2\left(D f(\gamma(t)) \frac{d \gamma}{d t}(t)\right)^{\prime} \frac{\partial^{2} \varphi}{\partial \bar{w} \partial w}(w(t)) D f(\gamma(t)) \frac{d \gamma}{d t}(t) \\
& +2 \operatorname{Re}\left[\left(\frac{\partial \varphi}{\partial w}(w(t))\right)^{\prime} D^{2} f(\gamma(t))\left(\frac{d \gamma}{d t}(t), \frac{d \gamma}{d t}(t)\right)\right] \\
& +2 \operatorname{Re}\left[\left(\frac{\partial \varphi}{\partial w}(w(t))\right)^{\prime} D f(\gamma(t)) \frac{d^{2} \gamma}{d t^{2}}(t)\right]
\end{aligned}
$$


where $w(t)=f(\gamma(t))$, for all $t \in(-\varepsilon, \varepsilon)$, so at $t=0$, we deduce that

$$
\begin{aligned}
& \operatorname{Re}\left\{\left(D f\left(z_{0}\right) v\right)^{\prime} \frac{\partial^{2} \varphi}{\partial w^{2}}\left(w_{0}\right) D f\left(z_{0}\right) v\right\} \\
+ & \left(\overline{D f\left(z_{0}\right) v}\right)^{\prime} \frac{\partial^{2} \varphi}{\partial \bar{w} \partial w}\left(w_{0}\right) D f\left(z_{0}\right) v \\
+ & \operatorname{Re}\left\{\left(\frac{\partial \varphi}{\partial w}\left(w_{0}\right)\right)^{\prime} D^{2} f\left(z_{0}\right)(v, v)+\left(\frac{\partial \varphi}{\partial w}\left(w_{0}\right)\right)^{\prime} D f\left(z_{0}\right) \frac{d^{2} \gamma}{d t^{2}}(0)\right\} \leq 0,
\end{aligned}
$$

where $w_{0}=f\left(z_{0}\right)$.

Let $\alpha:(-\varepsilon, \varepsilon) \rightarrow \mathbb{R}$ be given by $\alpha(t)=(\varphi \circ \gamma)(t)$ for all $t \in(-\varepsilon, \varepsilon)$. Since $\gamma(t) \in \partial B_{2 p}\left(r_{0}\right)$ when $t \in(-\varepsilon, \varepsilon)$, it follows that $\alpha(t)=r_{0}$ for all $t \in(-\varepsilon, \varepsilon)$, hence $\alpha^{\prime}(t) \equiv \alpha^{\prime \prime}(t) \equiv 0$ on $(-\varepsilon, \varepsilon)$.

Since

$$
\alpha^{\prime}(t)=2 \operatorname{Re}\left[\left(\frac{\partial \varphi}{\partial z}(\gamma(t))\right)^{\prime} \frac{d \gamma}{d t}(t)\right]
$$

and

$$
\begin{aligned}
\alpha^{\prime \prime}(t)= & 2\left\{\operatorname{Re}\left[\left(\frac{d \gamma}{d t}(t)\right)^{\prime} \frac{\partial^{2} \varphi}{\partial z^{2}}(\gamma(t)) \frac{d \gamma}{d t}(t)\right]\right. \\
& \left.+\left(\frac{d \gamma}{d t}(t)\right)^{\prime} \frac{\partial^{2} \varphi}{\partial \bar{z} \partial z}(\gamma(t)) \frac{d \gamma}{d t}(t)+\operatorname{Re}\left[\left(\frac{\partial \varphi}{\partial z}(\gamma(t))\right)^{\prime} \frac{d^{2} \gamma}{d t^{2}}(t)\right]\right\},
\end{aligned}
$$

for $t=0$ we deduce that

$$
0=\operatorname{Re}\left[\left(\frac{\partial \varphi}{\partial z}\left(z_{0}\right)\right)^{\prime} v\right]=p \operatorname{Re} \sum_{j=1}^{n}\left|z_{j}^{0}\right|^{2(p-1)} \bar{z}_{j}^{0} v_{j}
$$

and also

$$
\operatorname{Re}\left[\left(\frac{\partial \varphi}{\partial z}\left(z_{0}\right)\right)^{\prime} \frac{d^{2} \gamma}{d t^{2}}(0)\right]=-\operatorname{Re}\left[v^{\prime} \frac{\partial^{2} \varphi}{\partial z^{2}}\left(z_{0}\right) v\right]-\bar{v}^{\prime} \frac{\partial^{2} \varphi}{\partial \bar{z} \partial z}\left(z_{0}\right) v .
$$

Using (2.2), (2.10) and (2.11), we obtain

$$
\begin{aligned}
\operatorname{Re}\left\{\left(D f\left(z_{0}\right) v\right)^{\prime} \frac{\partial^{2} \varphi}{\partial w^{2}}\left(w_{0}\right) D f\left(z_{0}\right) v\right\}+\left(\overline{D f\left(z_{0}\right) v}\right)^{\prime} & \frac{\partial^{2} \varphi}{\partial \bar{w} \partial w}\left(w_{0}\right) D f\left(z_{0}\right) v \\
\leq & \frac{1}{s}\left\{\bar{v}^{\prime} \frac{\partial^{2} \varphi}{\partial \bar{z} \partial z}\left(z_{0}\right) v+\operatorname{Re}\left[v^{\prime} \frac{\partial^{2} \varphi}{\partial z^{2}}\left(z_{0}\right) v\right]\right. \\
& \left.-\operatorname{Re}\left[\left(\frac{\partial \varphi}{\partial z}\left(z_{0}\right)\right)^{\prime}\left[D f\left(z_{0}\right)\right]^{-1} D^{2} f\left(z_{0}\right)(v, v)\right]\right\},
\end{aligned}
$$

i.e. the relation (2.3). The proof is complete.

Remark 2.1. The result of Theorem 2.1 has the following geometric interpretation: since $f$ is locally biholomorphic at $z_{0}$, there exists a neigh- 
borhood $V$ of $z_{0}$ such that $f$ is biholomorphic on $V$. Then $f\left(\partial B_{2 p}\left(r_{0}\right) \cap V\right)$ is a real hypersurface in $\mathbb{C}^{n}$ and an outward normal vector at $w_{0}=f\left(z_{0}\right)$ is given by $N_{w_{0}}=\frac{\partial \phi}{\partial \bar{w}}\left(w_{0}\right)$, where $\phi(w)=\varphi\left(f^{-1}(w)\right)$, for all $w \in f(V)$, i.e.

$$
N_{w_{0}}=p\left(\overline{\left[D f\left(z_{0}\right)\right]^{-1}}\right)^{\prime}\left(\left|z_{1}^{0}\right|^{2(p-1)} z_{1}^{0}, \ldots,\left|z_{n}^{0}\right|^{2(p-1)} z_{n}^{0}\right)^{\prime} .
$$

Using (2.1), we deduce that the vectors $N_{w_{0}}$ and

$$
p\left(\left|f_{1}\left(z_{0}\right)\right|^{2(p-1)} f_{1}\left(z_{0}\right), \ldots,\left|f_{n}\left(z_{0}\right)\right|^{2(p-1)} f_{n}\left(z_{0}\right)\right)^{\prime}
$$

are collinear and have the same sense.

For $p=1$ in Theorem 2.1, we obtain the following result:

Corollary $2.1[\mathrm{KO} 4]$. Let $z_{0} \in B \backslash\{0\}$, and let $f \in H(B)$ be such that $f(0)=0, f$ is locally biholomorphic at $z_{0}$ and $f \not \equiv 0$. If

$$
\left\|f\left(z_{0}\right)\right\|=\max \left\{\|f(z)\|:\|z\| \leq\left\|z_{0}\right\|\right\},
$$

then there exists $0<s \leq\left\|z_{0}\right\|^{2} /\left\|f\left(z_{0}\right)\right\|^{2}$ such that

$$
\left(\left[D f\left(z_{0}\right)\right]^{-1}\right)^{\prime}\left(\bar{z}_{0}\right)=s \overline{f\left(z_{0}\right)}
$$

and

$$
\|v\|^{2}-\operatorname{Re}\left\{\bar{z}_{0}^{\prime}\left[D f\left(z_{0}\right)\right]^{-1} D^{2} f\left(z_{0}\right)(v, v)\right\} \geq s\left\|D f\left(z_{0}\right) v\right\|^{2},
$$

for all $v \in \mathbb{C}^{n} \backslash\{0\}$ with $\operatorname{Re}\left\langle z_{0}, v\right\rangle=0$.

Proof. In this case it is sufficient to put $\varphi(z)=\sum_{k=1}^{n}\left|z_{k}\right|^{2}$ in Theorem 2.1 for all $z=\left(z_{1}, \ldots, z_{n}\right)^{\prime} \in \mathbb{C}^{n}$. Then $\frac{\partial \varphi}{\partial z}(z)=\bar{z}, \frac{\partial^{2} \varphi}{\partial z^{2}}(z)=0$ (the null matrix) and $\frac{\partial^{2} \varphi}{\partial \bar{z} \partial z}(z)=I$, for all $z \in \mathbb{C}^{n}$, hence the asserted relations are immediate.

A direct consequence of Theorem 2.1 is given in the next result:

THEOREM 2.2. Let $f \in H\left(B_{2 p}\right)$, where $p \geq 1$, and let $g$ be a biholomorphic mapping on $B_{2 p}(r)$ for some $r>1$, with $f(0)=g(0)$. If there exist $z_{0} \in \partial B_{2 p}\left(r_{0}\right)$, with $0<r_{0}<1$, and $\zeta_{0} \in \partial B_{2 p}$ such that $f$ is locally biholomorphic at $z_{0}$,

$$
f\left(z_{0}\right)=g\left(\zeta_{0}\right) \quad \text { and } \quad f\left(B_{2 p}\left(r_{0}\right)\right) \subset g\left(B_{2 p}\right),
$$

then there exists $0<s \leq\left[\left\|z_{0}\right\|_{2 p}\right]^{2 p}$ such that the following relations hold at $z=z_{0}$ :

$$
\begin{aligned}
& \left(\left|z_{1}^{0}\right|^{2(p-1)} \bar{z}_{1}^{0}, \ldots,\left|z_{n}^{0}\right|^{2(p-1)} \bar{z}_{n}^{0}\right)\left[D f\left(z_{0}\right)\right]^{-1} \\
& \quad=s\left(\left|\zeta_{1}^{0}\right|^{2(p-1)} \bar{\zeta}_{1}^{0}, \ldots,\left|\zeta_{n}^{0}\right|^{2(p-1)} \bar{\zeta}_{n}^{0}\right)\left[D g\left(\zeta_{0}\right)\right]^{-1}
\end{aligned}
$$

and 
$(2.14)$

$$
\begin{aligned}
\operatorname{Re}\left\{w^{\prime} \frac{\partial^{2} \varphi}{\partial z^{2}}\left(\zeta_{0}\right) w\right\} & +\bar{w}^{\prime} \frac{\partial^{2} \varphi}{\partial \bar{z} \partial z}\left(\zeta_{0}\right) w \\
\leq & \frac{1}{s}\left\{\operatorname{Re}\left[v^{\prime} \frac{\partial^{2} \varphi}{\partial z^{2}}\left(z_{0}\right) v\right]+\bar{v}^{\prime} \frac{\partial^{2} \varphi}{\partial \bar{z} \partial z}\left(z_{0}\right) v\right. \\
& -\operatorname{Re}\left[\left(\frac{\partial \varphi}{\partial z}\left(z_{0}\right)\right)^{\prime}\left[D f\left(z_{0}\right)\right]^{-1} D^{2} f\left(z_{0}\right)(v, v)\right] \\
+ & \left.\operatorname{Re}\left[\left(\frac{\partial \varphi}{\partial z}\left(z_{0}\right)\right)^{\prime}\left[D f\left(z_{0}\right)\right]^{-1} D^{2} g\left(\zeta_{0}\right)(w, w)\right]\right\},
\end{aligned}
$$

for all $v \in \mathbb{C}^{n} \backslash\{0\}$ with $\operatorname{Re}\left\langle\frac{\partial \varphi}{\partial \bar{z}}\left(z_{0}\right), v\right\rangle=0$ and $w=\left[D g\left(\zeta_{0}\right)\right]^{-1} D f\left(z_{0}\right) v$, with $z_{0}=\left(z_{1}^{0}, \ldots, z_{n}^{0}\right)^{\prime}, \zeta_{0}=\left(\zeta_{1}^{0}, \ldots, \zeta_{n}^{0}\right)^{\prime}$ and $\varphi(z)=\sum_{k=1}^{n}\left|z_{k}\right|^{2 p}, z \in \mathbb{C}^{n}$.

Proof. Let $h: \bar{B}_{2 p}\left(r_{0}\right) \rightarrow \bar{B}_{2 p}$, and $h(z)=\left(g^{-1} \circ f\right)(z)$, for all $z \in$ $\bar{B}_{2 p}\left(r_{0}\right)$. Since $g$ is biholomorphic on $B_{2 p}(r)$, for some $r>1$, we have $h \in H\left(\bar{B}_{2 p}\left(r_{0}\right)\right), h(0)=0, h\left(z_{0}\right)=\zeta_{0}$, and from (2.12) we infer that $h\left(\bar{B}_{2 p}\left(r_{0}\right)\right) \subseteq \bar{B}_{2 p}$. Also, $h$ is locally biholomorphic at $z_{0}$, hence we deduce that

$$
1=\left\|h\left(z_{0}\right)\right\|_{2 p}=\max \left\{\|h(z)\|_{2 p}: z \in \bar{B}_{2 p}\left(r_{0}\right)\right\} .
$$

Using Theorem 2.1, there exists $0<s \leq\left[\left\|z_{0}\right\|_{2 p}\right]^{2 p}$ such that

$$
\begin{aligned}
\left(\left|z_{1}^{0}\right|^{2(p-1)} \bar{z}_{1}^{0}, \ldots,\left|z_{n}^{0}\right|^{2(p-1)} \bar{z}_{n}^{0}\right)\left[D h\left(z_{0}\right)\right]^{-1} & \\
& =s\left(\left|\zeta_{1}^{0}\right|^{2(p-1)} \bar{\zeta}_{1}^{0}, \ldots,\left|\zeta_{n}^{0}\right|^{2(p-1)} \bar{\zeta}_{n}^{0}\right)\left[D g\left(\zeta_{0}\right)\right]^{-1},
\end{aligned}
$$

i.e. the relation (2.13).

On the other hand, since $D f\left(z_{0}\right)=D g\left(\zeta_{0}\right) D h\left(z_{0}\right)$ and since $D^{2} f\left(z_{0}\right)$ is a continuous bilinear operator, we easily obtain

$$
\begin{aligned}
D^{2} f\left(z_{0}\right)(v, v)= & D^{2} f\left(\zeta_{0}\right)\left(D h\left(z_{0}\right) v, D h\left(z_{0}\right) v\right) \\
& +D g\left(\zeta_{0}\right) D^{2} h\left(z_{0}\right)(v, v)
\end{aligned}
$$

for all $v \in \mathbb{C}^{n}$. Multiplying both sides of (2.15) by

$$
p\left(\left|z_{1}^{0}\right|^{2(p-1)} \bar{z}_{1}^{0}, \ldots,\left|z_{n}^{0}\right|^{2(p-1)} \bar{z}_{n}^{0}\right)\left[D f\left(z_{0}\right)\right]^{-1}
$$

and using (2.13), we obtain

$$
\begin{aligned}
p\left(\left|z_{z^{0}}\right|^{2(p-1)} \bar{z}_{1}^{0}, \ldots,\left|z_{n}^{0}\right|^{2(p-1)} \bar{z}_{n}^{0}\right)\left[D f\left(z_{0}\right)\right]^{-1} D^{2} f\left(z_{0}\right)(v, v) & =p\left(\left|z_{1}^{0}\right|^{2(p-1)} \bar{z}_{1}^{0}, \ldots,\left|z_{n}^{0}\right|^{2(p-1)} \bar{z}_{n}^{0}\right)\left[D f\left(z_{0}\right)\right]^{-1} D^{2} g\left(\zeta_{0}\right)(w, w) \\
& +p\left(\left|z_{1}^{0}\right|^{2(p-1)} \bar{z}_{1}^{0}, \ldots,\left|z_{n}^{0}\right|^{2(p-1)} \bar{z}_{n}^{0}\right)\left[D h\left(z_{0}\right)\right]^{-1} D^{2} h\left(z_{0}\right)(v, v)
\end{aligned}
$$

where $w=D h\left(z_{0}\right) v$. From (2.16) we get 


$$
\begin{aligned}
\left(\frac{\partial \varphi}{\partial z}\left(z_{0}\right)\right)^{\prime}\left[D f\left(z_{0}\right)\right]^{-1} & D^{2} f\left(z_{0}\right)(v, v) \\
= & \left(\frac{\partial \varphi}{\partial z}\left(z_{0}\right)\right)^{\prime}\left[D f\left(z_{0}\right)\right]^{-1} D^{2} g\left(\zeta_{0}\right)(w, w) \\
& +\left(\frac{\partial \varphi}{\partial z}\left(z_{0}\right)\right)^{\prime}\left[D h\left(z_{0}\right)\right]^{-1} D^{2} h\left(z_{0}\right)(v, v) .
\end{aligned}
$$

Now, it is enough to take the real part in (2.17) and to use the relation (2.3) to obtain (2.14).

For the next result we use the following definition:

Definition 2.1. Let $f, g \in H\left(B_{2 p}\right)$. We say that $f$ is subordinate to $g$ (written $f \prec g$ ) if there exists $\omega \in H\left(B_{2 p}\right)$ such that $\omega(0)=0, \omega\left(B_{2 p}\right) \subset$ $B_{2 p}$ and $f(z)=(g \circ \omega)(z)$, for all $z \in B_{2 p}$.

Remark 2.2. It is clear that if $f \prec g$, then $f(0)=0$ and $f\left(B_{2 p}\right) \subset$ $g\left(B_{2 p}\right)$. But, if $g$ is biholomorphic on $B_{2 p}$, we deduce that $f \prec g$ iff $f(0)=$ $g(0)$ and $f\left(B_{2 p}\right) \subset g\left(B_{2 p}\right)$. Also, by a version of the Schwarz Lemma (see, for example, Theorem 1 in [GW] or Theorem 6 in [C], p. 55), if $f \prec g$, then $f\left(B_{2 p}(r)\right) \subset g\left(B_{2 p}(r)\right)$ for all $0<r<1$.

THEOREM 2.3. Let $f, g$ be locally biholomorphic mappings on $B_{2 p}$ and suppose that $g$ is biholomorphic on $B_{2 p}(r)$, for some $r>1$, and $f(0)=g(0)$. If $f$ is not subordinate to $g$, then there exist $0<r_{0}<1,0<s<1, z_{0} \in$ $\partial B_{2 p}\left(r_{0}\right)$ and $\zeta_{0} \in \partial B_{2 p}$ such that the relations (2.12)-(2.14) hold.

Proof. Since $f(0)=g(0)$ and $f \nprec g$, it follows that $f\left(B_{2 p}\right) \not \subset g\left(B_{2 p}\right)$. It is easy to see that there exists an $\varepsilon>0$ sufficiently small such that $f\left(B_{2 p}(\varepsilon)\right) \subset g\left(B_{2 p}\right)$. Hence, if we put

$$
r_{0}=\sup \left\{r>0: f\left(B_{2 p}(r)\right) \subset g\left(B_{2 p}\right)\right\},
$$

then $0<r_{0}<1$ and $f\left(\bar{B}_{2 p}\left(r_{0}\right)\right) \subseteq g\left(\bar{B}_{2 p}\right) \backslash g\left(B_{2 p}\right)$. So, we can find points $z_{0} \in \partial B_{2 p}\left(r_{0}\right)$ and $\zeta_{0} \in \partial B_{2 p}$ with $f\left(z_{0}\right)=g\left(\zeta_{0}\right)$. Now it is sufficient to apply Theorem 2.1 .

Definition 2.2. Let $\Omega \subseteq \mathbb{C}^{n} \times \mathbb{C}^{n}$ and $D \subseteq \mathbb{C}^{n}$ be two domains, and let $g$ be a biholomorphic mapping on $B_{2 p}(r)$, for some $r>1$, where $p \geq 1$. Suppose that $(g(0), 0) \in \Omega$ and $Q(g) \subset \Omega$, where

$$
Q(g)=\bigcup_{\substack{0<s<1 \\ \zeta \in \partial B_{2 p}}} Q_{s}(g, \zeta)
$$

and

$$
\begin{aligned}
Q_{s}(g, \zeta)=\{(u, v) & \in \mathbb{C}^{n} \times \mathbb{C}^{n}: u=g(\zeta), \\
& \left.v=s\left([D g(\zeta)]^{-1}\right)^{\prime}\left(\bar{\zeta}_{1}\left|\zeta_{1}\right|^{2(p-1)}, \ldots, \bar{\zeta}_{n}\left|\zeta_{n}\right|^{2(p-1)}\right)^{\prime}\right\},
\end{aligned}
$$


where $\zeta=\left(\zeta_{1}, \ldots, \zeta_{n}\right)^{\prime}$. The class $X_{2 p}(\Omega, D, g)$ consists of those mappings $\psi: \Omega \times B_{2 p} \rightarrow \mathbb{C}^{n}$ which satisfy the following conditions:

$$
\psi(g(0), 0 ; 0) \in D
$$

and

$$
\psi(u, v ; z) \notin D
$$

for all $(u, v) \in Q(g)$ and $z \in B_{2 p}$.

Using the above definition and Theorem 2.3, we obtain

THEOREM 2.4. Let $f$ be a locally biholomorphic mapping on $B_{2 p}$ and $g$ be a biholomorphic mapping on $B_{2 p}(r)$ for some $r>1$, with $f(0)=g(0)$. If

$$
\left(f(z),\left([D f(z)]^{-1}\right)^{\prime}\left(\bar{z}_{1}\left|z_{1}\right|^{2(p-1)}, \ldots, \bar{z}_{n}\left|z_{n}\right|^{2(p-1)}\right)^{\prime}\right) \in \Omega
$$

and if there exists a mapping $\psi \in X_{2 p}(\Omega, D, g)$ such that

$$
\psi\left(f(z),\left([D f(z)]^{-1}\right)^{\prime}\left(\bar{z}_{1}\left|z_{1}\right|^{2(p-1)}, \ldots, \bar{z}_{n}\left|z_{n}\right|^{2(p-1)}\right)^{\prime} ; z\right) \in D,
$$

for all $z \in B_{2 p}$, then $f \prec g$.

Proof. If the subordination $f \prec g$ does not hold, then, in view of Theorem 2.3, there exist $z_{0} \in B_{2 p}, \zeta_{0} \in \partial B_{2 p}$ and $0<s \leq\left[\left\|z_{0}\right\|_{2 p}\right]^{2 p}<1$ such that the relations (2.12)-(2.14) hold.

Let $u=f\left(z_{0}\right)$ and $v=\left(\left[D f\left(z_{0}\right)\right]^{-1}\right)^{\prime}\left(\bar{z}_{1}^{0}\left|z_{1}^{0}\right|^{2(p-1)}, \ldots, \bar{z}_{n}^{0}\left|z_{n}^{0}\right|^{2(p-1)}\right)^{\prime}$, where $z_{0}=\left(z_{1}^{0}, \ldots, z_{n}^{0}\right)^{\prime}$. Then $(u, v) \in Q_{s}\left(g, \zeta_{0}\right) \subseteq Q(g)$, hence, according to Definition 2.2, we deduce that $\psi\left(u, v ; z_{0}\right) \notin D$, contrary to the hypothesis. So, $f \prec g$.

We close this paper with the following applications. The first of them concerns a boundedness result; then we give some applications concerning subordinations by convex mappings defined on the Reinhardt domain $B_{2 p}$.

THEOREM 2.5. Let $f$ be a locally biholomorphic mapping on $B_{2 p}$ with $f(0)=0$, let $a$ and $b$ be two functions defined on $B_{2 p}$ with $|a(z)|-|b(z)| \geq 1$, for all $z \in B_{2 p}$. If

$$
\left\|a(z) f(z)+b(z)\left(\overline{[D f(z)]^{-1}}\right)^{\prime}\left(z_{1}\left|z_{1}\right|^{2(p-1)}, \ldots, z_{n}\left|z_{n}\right|^{2(p-1)}\right)^{\prime}\right\|_{2 p}<1,
$$

for all $z \in B_{2 p}$, then $\|f(z)\|_{2 p}<1$ on $B_{2 p}$.

Proof. Suppose that the inequality $\|f(z)\|_{2 p}<1$ does not hold at all points of $B_{2 p}$; then taking into account the fact that $f(0)=0$, we can easily find a real number $r \in(0,1)$ and $z_{0} \in B_{2 p} \backslash\{0\}$ such that

$$
\left\|f\left(z_{0}\right)\right\|_{2 p}=1=\max \left\{\|f(z)\|_{2 p}: z \in \bar{B}_{2 p}(r)\right\} .
$$


By Theorem 2.1, there exists $0<s \leq\left[\left\|z_{0}\right\|_{2 p}\right]^{2 p}$ such that

$$
\begin{aligned}
\left(\overline{\left[D f\left(z_{0}\right)\right]^{-1}}\right)^{\prime}\left(z_{1}^{0}\left|z_{1}^{0}\right|^{2(p-1)}, \ldots, z_{n}^{0}\left|z_{n}^{0}\right|^{2(p-1)}\right)^{\prime} & \\
& =s\left(f_{1}\left(z_{0}\right)\left|f_{1}\left(z_{0}\right)\right|^{2(p-1)}, \ldots, f_{n}\left(z_{0}\right)\left|f_{n}\left(z_{0}\right)\right|^{2(p-1)}\right)^{\prime},
\end{aligned}
$$

where $z_{0}=\left(z_{1}^{0}, \ldots, z_{n}^{0}\right)^{\prime}$. Hence, we obtain

$$
\begin{aligned}
& \left\|a\left(z_{0}\right) f\left(z_{0}\right)+b\left(z_{0}\right)\left(\overline{\left[D f\left(z_{0}\right)\right]^{-1}}\right)^{\prime}\left(z_{1}^{0}\left|z_{1}^{0}\right|^{2(p-1)}, \ldots, z_{n}^{0}\left|z_{1}^{0}\right|^{2(p-1)}\right)^{\prime}\right\|_{2 p} \\
& \quad=\left\|a\left(z_{0}\right) f\left(z_{0}\right)+s b\left(z_{0}\right)\left(f_{1}\left(z_{0}\right)\left|f_{1}\left(z_{0}\right)\right|^{2(p-1)}, \ldots, f_{n}\left(z_{0}\right)\left|f_{n}\left(z_{0}\right)\right|^{2(p-1)}\right)^{\prime}\right\|_{2 p} .
\end{aligned}
$$

Since $\|\cdot\|_{2 p}$ is a norm, the above equality implies

$$
\begin{aligned}
& \left\|a\left(z_{0}\right) f\left(z_{0}\right)+b\left(z_{0}\right)\left(\overline{\left[D f\left(z_{0}\right)\right]^{-1}}\right)^{\prime}\left(z_{1}^{0}\left|z_{1}^{0}\right|^{2(p-1)}, \ldots, z_{n}^{0}\left|z_{n}^{0}\right|^{2(p-1)}\right)^{\prime}\right\|_{2 p} \\
& \quad \geq\left|a\left(z_{0}\right)\right|-s\left|b\left(z_{0}\right)\right| \cdot\left\|\left(f_{1}\left(z_{0}\right)\left|f_{1}\left(z_{0}\right)\right|^{2(p-1)}, \ldots, f_{n}\left(z_{0}\right)\left|f_{n}\left(z_{0}\right)\right|^{2(p-1)}\right)^{\prime}\right\|_{2 p} .
\end{aligned}
$$

On the other hand, since $\left\|f\left(z_{0}\right)\right\|_{2 p}=1$, we deduce that

$$
\begin{aligned}
\|\left(f_{1}\left(z_{0}\right)\left|f_{1}\left(z_{0}\right)\right|^{2(p-1)}\right. & \left., \ldots, f_{n}\left(z_{0}\right)\left|f_{n}\left(z_{0}\right)\right|^{2(p-1)}\right)^{\prime} \|_{2 p}^{2 p} \\
= & \sum_{j=1}^{n}\left|f_{j}\left(z_{0}\right)\right|^{2 p}\left|f_{j}\left(z_{0}\right)\right|^{4 p(p-1)} \leq \sum_{j=1}^{n}\left|f_{j}\left(z_{0}\right)\right|^{2 p}=1,
\end{aligned}
$$

so

$$
\begin{aligned}
\left\|a\left(z_{0}\right) f\left(z_{0}\right)+b\left(z_{0}\right)\left(\overline{\left[D f\left(z_{0}\right)\right]^{-1}}\right)^{\prime}\left(z_{1}^{0}\left|z_{1}^{0}\right|^{2(p-1)}, \ldots, z_{n}^{0}\left|z_{n}^{0}\right|^{2(p-1)}\right)^{\prime}\right\|_{2 p} \\
\geq\left|a\left(z_{0}\right)\right|-s\left|b\left(z_{0}\right)\right| \geq\left|a\left(z_{0}\right)\right|-\left|b\left(z_{0}\right)\right| \geq 1 ;
\end{aligned}
$$

but this inequality contradicts the hypothesis.

Hence, $\|f(z)\|_{2 p}<1$, for all $z \in B_{2 p}$. The proof is complete.

THEOREM 2.6. Let $g$ be a biholomorphic mapping on $B_{2 p}(r)$, for some $r>1$, such that $g(0)=0$ and $g\left(B_{2 p}\right)$ is a convex domain in $\mathbb{C}^{n}$. Let $\phi$ be a holomorphic function on a domain $E \supset g\left(B_{2 p}\right)$ with $\operatorname{Re} \phi(g(z))>0$, for all $z \in B_{2 p}$. Also, let $A$ be a function defined on $B_{2 p}$ with $A(z) \geq 1$, for all $z \in B_{2 p}$. If $f$ is a locally biholomorphic mapping on $B_{2 p}$, with $f(0)=0$, $f\left(B_{2 p}\right) \subset E$ and if

$$
+\phi(f(z))\left(\overline{[D f(z)]^{-1}}\right)^{\prime}\left(z_{1}\left|z_{1}\right|^{2(p-1)}, \ldots, z_{n}\left|z_{n}\right|^{2(p-1)}\right)^{\prime} \in g\left(B_{2 p}\right),
$$

for all $z \in B_{2 p}$, then $f \prec g$.

Proof. If the subordination $f \prec g$ does not hold, then using Theorem 2.3, there exist $z_{0} \in B_{2 p} \backslash\{0\}, \zeta_{0} \in \partial B_{2 p}$ and $0<s<1$ such that $f\left(z_{0}\right)=g\left(\zeta_{0}\right)$ and

$$
\begin{aligned}
\left(\left|z_{1}^{0}\right|^{2(p-1)} \bar{z}_{1}^{0}, \ldots,\left|z_{n}^{0}\right|^{2(p-1)} \bar{z}_{n}^{0}\right)\left[D f\left(z_{0}\right)\right]^{-1} & \\
& =s\left(\left|\zeta_{1}^{0}\right|^{2(p-1)} \bar{\zeta}_{1}^{0}, \ldots,\left|\zeta_{n}^{0}\right|^{2(p-1)} \bar{\zeta}_{n}^{0}\right)\left[D g\left(\zeta_{0}\right)\right]^{-1},
\end{aligned}
$$

where $z_{0}=\left(z_{1}^{0}, \ldots, z_{n}^{0}\right)^{\prime}$ and $\zeta_{0}=\left(\zeta_{1}^{0}, \ldots, \zeta_{n}^{0}\right)^{\prime}$. 
Let

$\psi(z)=A(z) f(z)+\phi(f(z))\left(\overline{[D f(z)]^{-1}}\right)^{\prime}\left(z_{1}\left|z_{1}\right|^{2(p-1)}, \ldots, z_{n}\left|z_{n}\right|^{2(p-1)}\right)^{\prime} ;$

then at $z=z_{0}$ we obtain

(2.21) $\psi\left(z_{0}\right)=A\left(z_{0}\right) g\left(\zeta_{0}\right)$

$$
+s \phi\left(g\left(\zeta_{0}\right)\right)\left(\overline{\left[D g\left(\zeta_{0}\right)\right]^{-1}}\right)^{\prime}\left(\zeta_{1}^{0}\left|\zeta_{1}^{0}\right|^{2(p-1)}, \ldots, \zeta_{n}^{0}\left|\zeta_{n}^{0}\right|^{2(p-1)}\right)^{\prime} .
$$

Since $g\left(B_{2 p}\right)$ is a convex domain, hence starlike with respect to zero, and $g\left(\partial B_{2 p}\right)$ is a real hypersurface in $\mathbb{C}^{n}$, it follows that $g\left(\partial B_{2 p}\right)$ is a starlike hypersurface, so the following relation holds (see for example Theorem 2 of [GW] or Theorem 4 of [S1]):

$$
\operatorname{Re}\left\langle g\left(\zeta_{0}\right), N_{w_{0}}\right\rangle \geq 0,
$$

where $w_{0}=g\left(\zeta_{0}\right)$ and $N_{w_{0}}$ is the outward normal vector to $g\left(\partial B_{2 p}\right)$ at $w_{0}$. But

$$
N_{w_{0}}=p\left(\overline{\left(D g\left(\zeta_{0}\right)\right]^{-1}}\right)^{\prime}\left(\zeta_{1}^{0}\left|\zeta_{1}^{0}\right|^{2(p-1)}, \ldots, \zeta_{n}^{0}\left|\zeta_{n}^{0}\right|^{2(p-1)}\right)^{\prime},
$$

hence, using the relations (2.21) and (2.22), we deduce that

$$
\begin{aligned}
\operatorname{Re}\left\langle\psi\left(z_{0}\right)-g\left(\zeta_{0}\right), N_{w_{0}}\right\rangle= & \left(A\left(z_{0}\right)-1\right) \operatorname{Re}\left\langle g\left(\zeta_{0}\right), N_{w_{0}}\right\rangle \\
& +(s / p) \operatorname{Re} \phi\left(g\left(\zeta_{0}\right)\right)\left\|N_{w_{0}}\right\|^{2} .
\end{aligned}
$$

Now, from the hypothesis, we conclude that

$$
\operatorname{Re}\left\langle\psi\left(z_{0}\right)-g\left(\zeta_{0}\right), N_{w_{0}}\right\rangle \geq 0 .
$$

On the other hand, since $g\left(B_{2 p}\right)$ is a convex domain, the above relation implies $\psi\left(z_{0}\right) \notin g\left(B_{2 p}\right)$, contrary to $(2.20)$. The proof is complete.

For $g(z)=z$ in Theorem 2.6, we obtain the following result:

Corollary 2.2. Let $A$ be a function defined on $B_{2 p}$ such that $A(z) \geq 1$, for all $z \in B_{2 p}$. Let $\phi$ be a holomorphic function defined on a domain $E \supset$ $B_{2 p}$ such that $\operatorname{Re} \phi(z)>0$ for all $z \in B_{2 p}$. If $f$ is a locally biholomorphic mapping defined on $B_{2 p}$ with $f(0)=0, f\left(B_{2 p}\right) \subset E$, and if

$$
\left\|A(z) f(z)+\phi(f(z))\left(\overline{[D f(z)]^{-1}}\right)^{\prime}\left(z_{1}\left|z_{1}\right|^{2(p-1)}, \ldots, z_{n}\left|z_{n}\right|^{2(p-1)}\right)^{\prime}\right\|_{2 p}<1
$$

for all $z \in B_{2 p}$, then $\|f(z)\|_{2 p}<1$ on $B_{2 p}$.

Another interesting result is obtained from Theorem 2.5 if we let $p=1$.

COROLlary 2.3. Let $g$ be a biholomorphic mapping on $\bar{B}$ such that $g(0)=0$ and $g\left(B_{2 p}\right)$ is a convex domain in $\mathbb{C}^{n}$, let $\phi$ be a holomorphic function defined on a domain $E \supset g(B)$ with $\operatorname{Re} \phi(g(z))>0$, for all $z \in B$, and let $A$ be a function defined on $B$ with $A(z) \geq 1$, for all $z \in B$. If $f \in H(B)$ is locally biholomorphic on $B, f(0)=0$, and if

$$
A(z) f(z)+\phi(f(z))\left(\overline{[D f(z)]^{-1}}\right)^{\prime} z \in g(B),
$$

for all $z \in B$, then $f \prec g$. 


\section{References}

[C] B. Chabat, Introduction à l'analyse complexe, tome II, Mir, Moscou, 1990.

[GW] S. Gong and S. K. Wang, A necessary and sufficient condition that biholomorphic mappings are starlike on a class of Reinhardt domains, Chinese Ann. Math. Ser. B 13 (1) (1992), 95-104.

[GWQ] S. Gong, S. K. Wang and Q. Yu, Biholomorphic convex mappings of ball in $\mathbb{C}^{n}$, Pacific J. Math. 161 (1993), 287-306.

[K] K. Kikuchi, Starlike and convex mappings in several complex variables, ibid. 44 (1973), 569-580.

[KO1] G. Kohr, On some partial differential subordinations for holomorphic mappings in $\mathbb{C}^{n}$, Libertas Math. 115 (1996), 129-142.

[KO2] G. Kohr and M. Kohr-Ile, Partial differential subordinations for holomorphic mappings of several complex variables, Studia Univ. Babeş-Bolyai Math. 60 (4) (1995), 46-62.

[KO3] G. Kohr and P. Liczberski, General partial differential subordinations for holomorphic mappings in $\mathbb{C}^{n}$, Math. Nachr., to appear.

[KO4] G. Kohr and C. Pintea, An extension of Jack-Miller-Mocanu's Lemma for holomorphic mappings defined on some domains in $\mathbb{C}^{n}$, to appear.

[L] P. Liczberski, Jack's Lemma for holomorphic mappings in $\mathbb{C}^{n}$, Ann. Univ. Mariae Curie-Skłodowska Sect. A 40 (1986), 131-140.

[MM1] S. S. Miller and P. T. Mocanu, Differential subordinations and inequalities in the complex plane, J. Math. Anal. Appl. 65 (1978), 289-305.

[MM2] -, -, Differential subordinations and inequalities in the complex plane, J. Differential Equations 67 (1987), 199-211.

[S1] T. J. Suffridge, The principle of subordination applied to functions of several variables, Pacific J. Math. 33 (1970), 241-248.

[S2] —, Starlikeness, convexity and other geometric properties of holomorphic maps in higher dimensions, in: Lecture Notes in Math. 599, Springer, 1976, 146-159.

Faculty of Mathematics

Babeş-Bolyai University

1 M. Kogălniceanu Str.

3400 Cluj-Napoca, Romania

E-mail: gkohr@math.ubbcluj.ro 